\section{A influência das informações da Pesquisa Nacional de Saúde sobre a estimativa atual e a trajetória do aleitamento materno exclusivo no Brasil}

\section{The influence of information from the Brazilian National Health Survey on the current estimate and trend in exclusive breastfeeding in Brazil}

La influencia de la información de la Encuesta Nacional de Salud acerca de la estimación actual y trayectoria de la lactancia materna exclusiva en Brasil

\section{Resumo}

Nosso propósito foi discutir a consistência da estimativa dos indicadores do aleitamento materno na Pesquisa Nacional de Saúde (PNS) e suas implicações para a análise da trajetória destes indicadores entre 1986 e 2013. A principal limitação para a estimativa atual (2013) dos indicadores de aleitamento materno com base na PNS e sua trajetória (1986-2013) é a ausência da data de entrevista nos microdados, comprometendo o cálculo exato da idade. Estudos prévios mostraram cenários distintos do aleitamento materno exclusivo (AME) baseando-se na PNS: 36,6\% (estabilização da prevalência em comparação com 2006 [Pesquisa Nacional de Demografia e Saúde PNDS]) e 20,3\% (retrocesso da prevalência). Simulações de data da entrevista utilizando data da coleta de dados da PNS (agosto/2013 a fevereiro/2014) nos indicaram variação de prevalência de 25,3\% a 42,8\% (AME); 54\% a 61,5\% (aleitamento materno) e 32,7 a 40,5\% (aleitamento materno), respectivamente. A divergência de cenário apresentado no status do aleitamento materno nos desperta para a inviabilidade de estimativa do AME em 2013 na ausência de esclarecimento da data da entrevista.

Aleitamento Materno; Indicadores (Estatísticas); Inquéritos Epidemiológicos
COMUNICAÇÃO BREVE

BRIEF COMMUNICATION
Ana Elisa Madalena Rinaldi 1

Wolney Lisboa Conde 2

doi: 10.1590/0102-311X00190118

\author{
Correspondência \\ A. E. M. Rinaldi \\ Faculdade de Medicina, Universidade Federal de Uberlândia. \\ Rua Pará 1720, 2U, Uberlândia, MG 38402-022, Brasil. \\ anaelisarinaldi@gmail.com \\ 1 Universidade Federal de Uberlândia, Uberlândia, Brasil. \\ 2 Faculdade de Saúde Pública, Universidade de São Paulo, \\ São Paulo, Brasil.
}




\section{Introdução}

A série histórica com estimativas nacionais dos indicadores do aleitamento materno para crianças menores de dois anos está baseada nas Pesquisas Nacional de Demografia e Saúde de 1986 1, 19962 e 20063 , permitindo análises consistentes com trajetória positiva e otimista ${ }^{4}$. Posteriormente, as estimativas podem ser obtidas valendo-se da II Pesquisa de Prevalência de Aleitamento Materno nos municípios brasileiros (II PPAM) 5, realizada em 266 municípios, incluindo capitais, cuja população-alvo foram crianças menores de 12 meses selecionadas por amostra probabilística em postos de vacinação em dias de Campanha Nacional de Imunização. O último dado nacional dos indicadores é proveniente da Pesquisa Nacional de Saúde (PNS) 6.

A informação da idade exata da criança é fundamental para o cálculo dos indicadores do aleitamento materno pela especificidade das recomendações. Dessa forma, nossa proposta é discutir a consistência da estimativa dos indicadores do aleitamento materno na PNS (2013) e suas implicações para a análise da trajetória destes indicadores entre 1986 e 2013.

Estruturamos nosso comentário em dois pontos de questionamento: (1) prevalência dos indicadores do aleitamento materno com base nos dados da PNS (2013) em função da data de referência para a inclusão das crianças menores de 24 meses; e (2) trajetória das prevalências dos indicadores de aleitamento materno com base nas pesquisas realizadas antes de 2013 e sua comparação com os dados da PNS.

\section{1o ponto: consistência da prevalência do aleitamento materno em 2013}

Baseando-se nos microdados da PNS 6, surge o primeiro questionamento que é a ausência da data da entrevista. A informação etária está agrupada nas faixas “0-11,9 meses” e "12,0-23,9 meses”, impossibilitando calcular os indicadores recomendados pela Organização Mundial da Saúde (OMS), principalmente do aleitamento materno exclusivo (AME).

Nos manuais metodológicos da PNS, as crianças consideradas elegíveis para compor a amostra foram aquelas nascidas entre 28 de julho de 2011 e 27 de julho de 2013 . Entretanto, o campo da pesquisa foi realizado no período de agosto/2013 a fevereiro/2014 8 . Essas duas informações indicam o intervalo de 1 a 7 meses entre a coleta e a última data de referência (27 de julho de 2013). Em função da ausência da data da entrevista, decisões metodológicas distintas foram adotadas para que fosse possível o cálculo dos indicadores do aleitamento materno. Bocollini et al. 9 utilizaram as informações disponíveis no DATASUS (Departamento de Informática do SUS) sem descrever a data da entrevista adotada, e Flores et al. 10 calcularam a idade usando a data "27 de julho de 2013". Com base nessas decisões, podemos observar diferença de 4 pontos percentuais para aleitamento materno $(52,1 \% 9$ vs. $56 \% 10)$ e 16 pontos percentuais para $\operatorname{AME}(36,6 \% 9$ e $20,6 \% 10)$.

Diante da divergência para AME, acreditamos que a estimativa mais viável seja a apresentada por Bocollini et al. ${ }^{9}$, pois houve aumento da prevalência do AME já documentada entre 2006 e 2008 (38,6 para 41\%) 4 . Como contraste, apresentamos aqui a simulação das possíveis prevalências dos indicadores AME, expresso nas faixas < 6 meses; 0-2 meses; 3-5 meses; aleitamento materno e aleitamento materno continuado aos 2 anos, em função de diferentes datas de referência para a coleta de dados 7 (Tabela 1). Todas as análises foram baseadas nas crianças que tiveram suas informações respondidas pela mãe/responsável ( $81 \%$ da amostra) 9 e foram realizadas considerando a estrutura complexa da amostra e fator de ponderação amostral.

$\mathrm{Na}$ simulação, inicialmente, foi calculada a idade das crianças utilizando-se a data de nascimento (disponível nos microdados da PNS) e oito possíveis datas de entrevistas, correspondentes aos meses da coleta de dados (julho/2013 a fevereiro/2014) 8. Por compatibilidade com a data de referência da coleta da PNS, em cada mês foi selecionado o dia 27. Posteriormente, as prevalências dos indicadores do aleitamento materno foram estimadas utilizando-se essas datas de entrevista como referência para o cálculo da idade (DE1 a DE8) (Tabela 1). O intuito dessa simulação foi estimar os efeitos nas prevalências dos indicadores segundo diferentes períodos de coleta de dados, uma vez que há divergência entre o período de referência para a inclusão de crianças menores de 24 meses ( 28 de julho de 2011 a 27 de julho de 2013) e o campo da pesquisa (agosto/2013 a fevereiro/2014). 


\section{Tabela 1}

Descrição dos indicadores do aleitamento materno com base em diferentes datas de entrevista, considerando-se somente as crianças cujas mães responderam às questões da Pesquisa Nacional de Saúde (L020 = 1) (base = 4.215), 2013.

\begin{tabular}{|c|c|c|c|c|c|c|c|c|c|}
\hline Indicador & Boccolini et al. 9 & DE1 & DE2 & DE3 & DE4 & DE5 & DE6 & DE7 & DE8 \\
\hline Aleitamento materno & 52,1 & 52,9 & 54,0 & 54,8 & 55,5 & 55,9 & 57,2 & 59,4 & 61,5 \\
\hline Aleitamento materno 2 anos & 31,8 & 27,9 & 32,7 & 33,1 & 33,1 & 33,8 & 33,9 & 37,6 & 40,5 \\
\hline AME & 36,6 & 20,8 & 25,3 & 29,8 & 33,9 & 38,0 & 42,8 & $\star \star \star$ & $\star \star \star$ \\
\hline $\operatorname{AME}(0-2)$ * & 49,7 & 33,6 & 38,6 & 42,8 & $\star \star \star$ & $\star \star \star$ & $\star \star \star$ & $\star \star \star$ & $\star \star \star$ \\
\hline $\operatorname{AME}(3-5) * *$ & 14,6 & 7,8 & 15,3 & 24,5 & 32,3 & 36,8 & 39,9 & $\star \star \star$ & $\star \star \star$ \\
\hline
\end{tabular}

Aleitamento materno: < 24 meses; aleitamento materno 2 anos: aleitamento continuado para crianças de 20-23 meses; AME: aleitamento materno exclusivo; DE: data da entrevista; DE1: 27 de julho de 2013; DE2: 27 de agosto de 2013; DE3: 27 de setembro de 2013; DE4: 27 de outubro de 2013; DE5: 27 de novembro de 2013; DE6: 27 de dezembro de 2013; DE7: 27 de janeiro de 2014; DE8: 27 de fevereiro de 2014.

* AME (0-2 meses): prevalência de aleitamento exclusivo entre crianças de 0-2 meses completos;

** AME (3-5 meses): prevalência de aleitamento exclusivo entre crianças de 0-5 meses completos;

*** Ausência de crianças nestas faixas etárias em função da data de entrevista simulada.

As estimativas de AME apresentaram as maiores discrepâncias no período simulado, com variação de 22 pontos percentuais $(20,8$ a 42,8\%) para faixa < 6 meses; 9,2 pontos percentuais para faixa de 0-2 meses e 32,1 pontos percentuais para 3-5 meses (Tabela 1). A estimativa obtida por Boccolini et al. 9 foi semelhante àquelas que obteríamos caso nossa data de referência para pesquisa fosse novembro/2013. A prevalência do AME é a que apresenta maior variação por incluir as crianças mais novas e que seriam mais afetadas pela imprecisão da data da entrevista. A aplicação da data de 27 de julho de 2013 possivelmente leva à subestimação das prevalências dos indicadores por modificar a distribuição etária da base de dados das crianças incluídas, resultando em menor percentual de crianças menores de 6 meses.

A questão central é que os três textos, incluindo este, não estão trabalhando com o mesmo denominador em função de decisões metodológicas. Neste comentário descrevemos e fundamentamos cada decisão ou etapa do cálculo para eliminar ambiguidades que possam impedir a apreciação das decisões tomadas.

\section{0 ponto: consistência da trajetória do AME entre 1986 e 2013}

A análise da trajetória dos indicadores do aleitamento materno nos aproximaria de conclusões mais consistentes (Figura 1). A trajetória das prevalências foi realizada com dados de AM provenientes de cinco pesquisas de âmbito nacional: PNDS 1986, 1996, 2006; II PPAM e PNS. Ao considerarmos a estimativa de Flores et al. 10, concluiríamos que a prevalência de AME reduziu quase pela metade (20,8\%) entre 2006 e 2013. Já a prevalência apresentada por Boccolini et al. ${ }^{9}$ nos parece mais plausível principalmente pela tendência de aumento da prevalência de AME entre 2006 e 2008 (Figura 1), semelhante aos dados publicados anteriormente 4 e continuidade das ações pró-aleitamento materno no país (Figura 2). Adicionalmente, adotando como ponto de partida o mesmo incremento da prevalência de AME ocorrido entre 1996 e 2006 (1,03\%/ano) para 2006 e 2013 com ajuste para sete anos, a projeção da prevalência de AME em 2013 alcançaria 46,7\%.

Destacamos que a comparação das estimativas entre as PNDS 1986-2006 e II PPAM deve ser realizada com cautela no que tange principalmente às diferenças do desenho amostral. Entretanto, as questões sobre aleitamento são semelhantes entre os inquéritos 4 e, independentemente da precisão das estimativas em relação ao espaço nacional, é provável que a variabilidade do fenômeno tenha sido bem capturada no desenho amostral realizado.

Ao analisarmos o conjunto dos três indicadores de aleitamento materno utilizando-se a data de 27 de julho de 2013, enfrentamos três cenários aparentemente discrepantes: redução do AME (40,5\% 
Figura 1

Trajetória das prevalências do AME (1a), aleitamento materno aos 2 anos (1b) e do aleitamento materno (1c) com base na Pesquisa Nacional de Demografia e Saúde $(1986,1996,2006)$ e na Pesquisa Nacional de Saúde (datas simuladas).

1a) Prevalência do AME (0-5,9 meses)

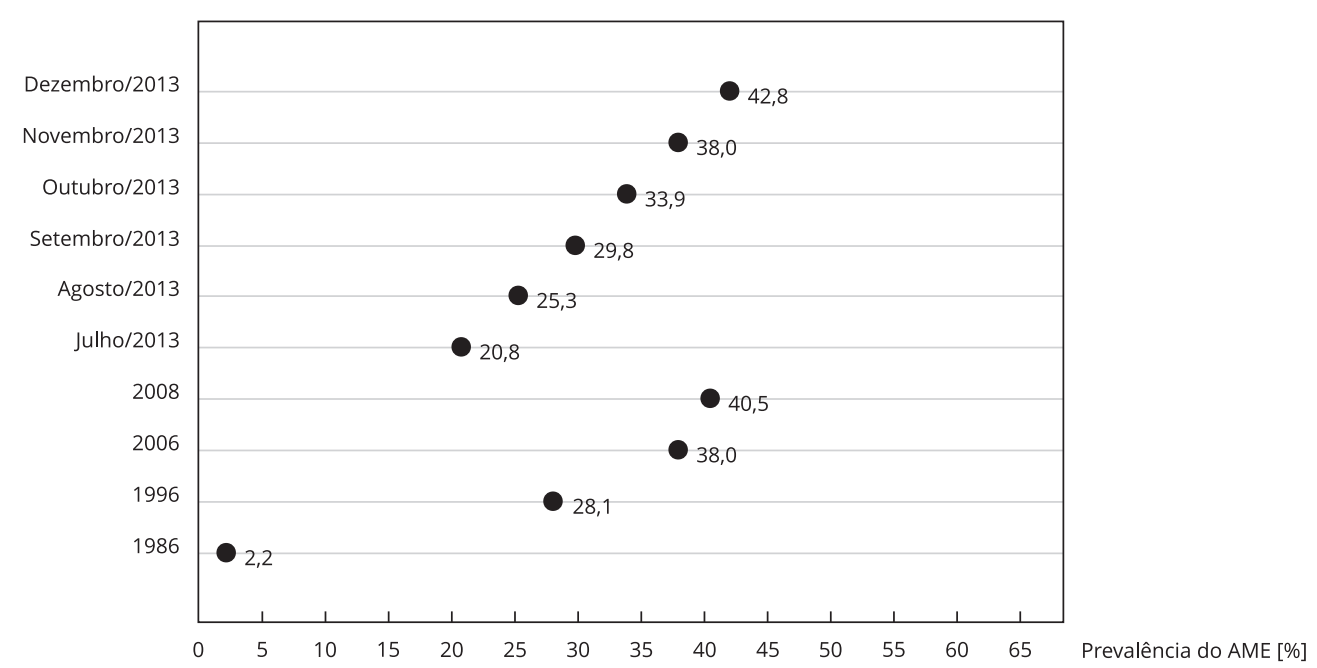

1b) Prevalência do aleitamento materno - 24 meses (20-23 meses)

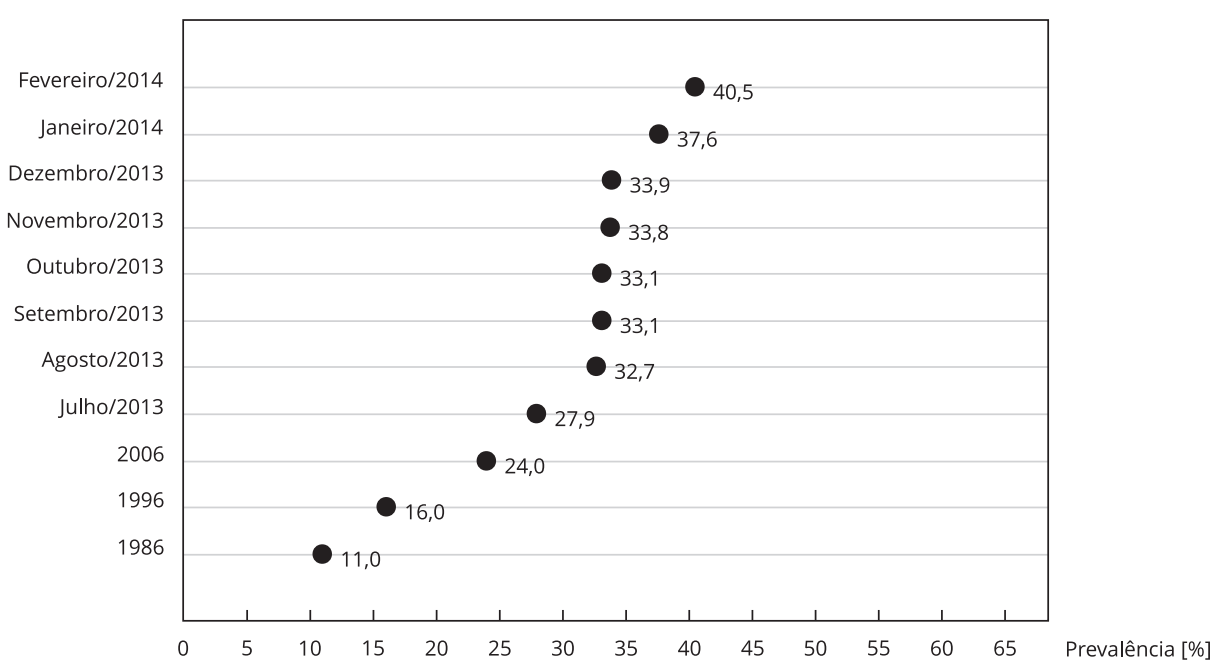

(continua)

em 2008 e 20,8\% em 2013), aumento do aleitamento materno continuado aos 2 anos (24\% em 2006 e $29,7 \%$ em 2013) e redução do aleitamento materno (56,7\% para 52,9\%). Parece-nos inconsistente observar aumento do aleitamento materno continuado aos 2 anos e queda no aleitamento materno, pois espera-se que ambos aumentem. Uma possível explicação é que no denominador do indicador aleitamento materno também foram contempladas as crianças menores de 6 meses, faixa etária que parece apresentar o maior efeito da indefinição da data da entrevista. 


\section{Figura 1 (continuação)}

1c) Prevalência do aleitamento materno (0-23,9 meses)

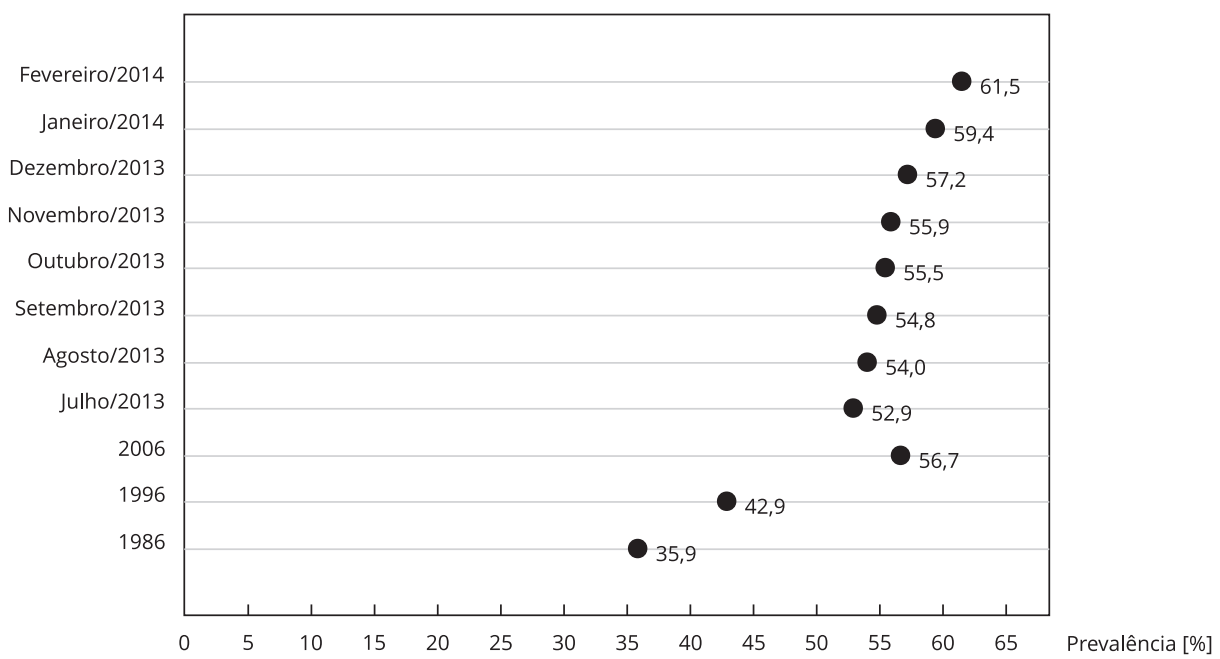

Aleitamento materno: < 24 meses; aleitamento materno aos 2 anos: aleitamento continuado para crianças de 20-23 meses; AME: aleitamento materno exclusivo.

\section{Figura 2}

Cronologia das ações de promoção, apoio e proteção ao aleitamento materno realizadas no Brasil, $1980-2018$.

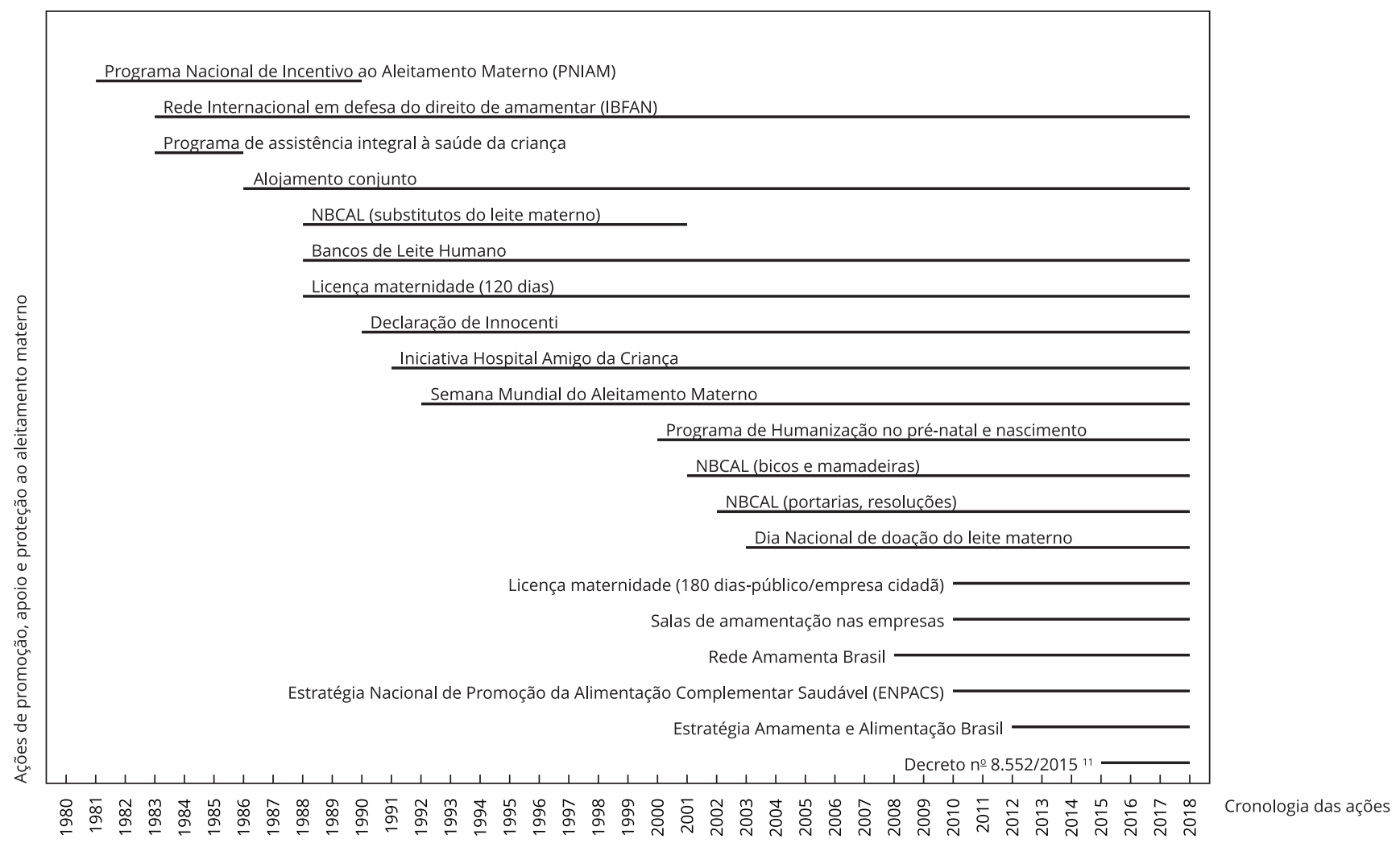

NBCAL: Norma Brasileira de Comercialização de Alimentos para Lactantes e Crianças da Primeira Infância, Bicos, Chupetas e Mamadeiras. 
Na estimativa de 1996 ocorreu uma provável superestimação do AME em função do fluxo de questões de alimentação no questionário: crianças cujas mães declararam estar amamentando exclusivamente não foram questionadas sobre outros alimentos oferecidos no dia anterior 9 .

\section{Considerações finais}

Baseando-se nesse questionamento específico sobre o panorama relativamente incerto dos indicadores, especialmente o AME, fechamos este comentário com duas considerações principais sobre organização das pesquisas de saúde no Brasil. A primeira é a necessidade de consolidação da PNS como pesquisa efetiva para analisar o perfil de saúde da população brasileira. Por ser a única pesquisa de saúde nacional, seria relevante incluir também crianças e adolescentes. Nessa primeira edição da PNS foram incluídas as crianças menores de 24 meses, entretanto, no aspecto específico do aleitamento materno pode-se identificar a presença dos indicadores descolados dos seus contextos sociodemográficos e de saúde. Outra consideração é a viabilidade da continuidade das pesquisas de demografia e saúde no Brasil com intervalo de 10 anos, pois a análise da efetividade das ações exige dados atualizados. O Estudo Nacional de Alimentação e Nutrição Infantil (https://enani.nutricao.ufrj.br/), iniciado em 2019, possibilitará a análise mais robusta dessa trajetória dos indicadores do aleitamento materno no Brasil.

Em qualquer dos cenários aqui considerados, a trajetória crescente do aleitamento materno no Brasil está abaixo de seu potencial se levarmos em consideração o conjunto de profissionais envolvidos e o arco de ações desenvolvidas. Outro aspecto relevante é a coleta e disponibilidade de informações mais amplas que incluam variáveis comportamentais, visões de mundo de mães/responsáveis pelas crianças, informações sobre protocolos efetivados na atenção básica, percepções pelos pacientes das condutas e recomendações dos profissionais de saúde envolvidos no seu atendimento, segurança nos campos legal e trabalhista ao longo da gravidez e amamentação. Essa massa de informações permitirá análises mais sofisticadas em amplos conjuntos populacionais sobre os vetores macro e micro que impulsionam ou dificultam a decisão de amamentar e sua implementação. Tais análises irão refinar intervenções voltadas para a promoção e apoio ao aleitamento materno mais efetivas, dirigidas a perfis vulneráveis. 


\section{Colaboradores}

A. E. M. Rinaldi contribuiu na elaboração do manuscrito, análise dos dados, redação e revisão final. W. L. Conde contribuiu na análise dos dados e revisão final.

\section{Informações adicionais}

ORCID: Ana Elisa Madalena Rinaldi (0000-00030154-554X); Wolney Lisboa Conde (0000-00030493-134X).

\section{Referências}

1. Arruda JM, Rutenberg N, Morris L, Ferraz EA. Pesquisa Nacional sobre Saúde Materno-Infantil e Planejamento Familiar - PNSMIPF Brasil, 1986. Rio de Janeiro: Sociedade Civil Bem-Estar Familiar no Brasil; 1987.

2. Sociedade Civil Bem-Estar Familiar no Brasil; Macro International. Pesquisa Nacional sobre Demografia e Saúde - 1996. Rio de Janeiro: Sociedade Civil Bem-Estar Familiar no Brasil/ DHS Program Macro International; 1997.

3. Centro Brasileiro de Análise e Planejamento. Pesquisa Nacional de Demografia e Saúde da Criança e da Mulher. PNDS. 2006. Brasília: Ministério da Saúde; 2009. (Série G. Estatística e Informação em Saúde).

4. Venancio SI, Saldiva SRDM, Monteiro CA. Tendência secular da amamentação no Brasil. Rev Saúde Pública 2013; 47:1205-8.

5. Departamento de Ações Programáticas e Estratégicas, Secretaria de Atenção à Saúde, Ministério da Saúde. II Pesquisa de Prevalência de Aleitamento Materno em Municípios Brasileiros. Brasília: Ministério da Saúde; 2008. (Série C. Projetos, Programas e Relatórios).

6. Instituto Brasileiro de Geografia e Estatística. Pesquisa Nacional de Saúde. Microdados. https://ww2.ibge.gov.br/home/estatistica/po pulacao/pns/2013/default_microdados.shtm (acessado em 20/Jul/2018).

7. Ministério da Saúde. Informações de Saúde (TABNET) - Inquéritos e Pesquisas. Nota técnica: Pesquisa Nacional de Saúde - Módulo de crianças com menos de dois anos. http:// tabnet.datasus.gov.br/cgi/pns/Notas_Tecni cas_PNS_L.pdf (acessado em 20/Jul/2018).

8. Damacena GN, Szwarcwald CL, Malta DC, Souza-Junior, PRB, Vieira MLFP, Pereira CA, et al. O processo de desenvolvimento da Pesquisa Nacional de Saúde no Brasil, 2013. Epidemiol Serv Saúde 2015; 24:197-206.

9. Boccolini DC, Boccolini PMM, Monteiro FR, Venâncio SI, Giugliani ERJ. Breastfeeding indicadors trends in Brazil for three decades. Rev Saúde Pública 2017; 51:108.

10. Flores TR, Nunes BP, Neves RG, Wendt AT, Costa CS, Wehrmeister FC, et al. Consumo de leite materno e fatores associados em crianças menores de dois anos: Pesquisa Nacional de Saúde, 2013. Cad Saúde Pública 2017; 33:e00068816.

11. Brasil. Decreto no 8.552 de 03 de novembro de 2015. Regulamenta a Lei no 11.265 , de 3 de janeiro de 2006, que dispõe sobre a comercialização de alimentos para lactentes e crianças de primeira infância e de produtos de puericultura correlatos. Diário Oficial da União 2015; 4 nov. 


\section{Abstract}

The study aimed to discuss the consistency of estimates of maternal breastfeeding in the Brazilian National Health Survey (PNS) and the implications for the analysis of trends in these indicators from 1986 to 2013. The main limitation for the current estimate (2013) of breastfeeding indicators based on the PNS and their trend (1986-2013) is the absence of the interview date in the microdata, thus hindering a precise calculation of age. Previous studies have shown different scenarios for exclusive breastfeeding based on the PNS: $36.6 \%$ (stabilization of prevalence in comparison to 2006 [Brazilian National Survey of Demography and Health - PNDSJ) and 20.3\% (downward prevalence). Simulations of the interview date using the date of data collection in the PNS (August 2013 to February 2014) indicated a variation of prevalence $25.3 \%$ to $42.8 \%$ (exclusive breastfeeding); $54 \%$ to $61.5 \%$ (breastfeeding); and $32.7 \%$ to 40.5\% (breastfeeding, second), respectively. The discrepancy in the scenario for breastfeeding status calls attention to the unfeasibility of the exclusive breastfeeding estimate for 2013, pending clarification of the interview date.

Breast Feeding; Indicators (Statistics); Health Surveys

\section{Resumen}

Nuestro objetivo fue discutir la consistencia de la estimación de los indicadores de lactancia materna en la Encuesta Nacional de Salud (ENS) y sus implicaciones para el análisis de la trayectoria de estos indicadores entre 1986 y 2013. La principal limitación para la estimación actual (2013) de los indicadores de lactancia materna, basados en la ENS y su trayectoria (1986-2013), es la ausencia de fecha de entrevista en los microdatos, comprometiendo el cálculo exacto de la edad. Estudios previos mostraron escenarios distintos del lactancia materna exclusiva (LME) basándose en la ENS: 36,6\% (estabilización de la prevalencia, en comparación con 2006 [Encuesta Nacional de Demografía y Salud - ENDS]) y 20,3\% (retroceso de la prevalencia). Simulaciones con fecha de la entrevista, utilizando la fecha de recogida de datos de la ENS (agosto/2013 a febrero/2014), nos indicaron una variación de prevalencia entre un $25,3 \%$ a un $42,8 \%$ (LME); de un $54 \%$ a un $61,5 \%$ (lactancia materna) y de 32,7 a un 40,5\% (lactancia materna), respectivamente. La divergencia de escenario, presentado en el status del lactancia materna, nos expone la inviabilidad de la estimación de la LME en 2013, debido a la ausencia de información respecto a la fecha de la entrevista.

Lactancia Materna; Indicadores (Estadística);

Encuestas Epidemiológicas
Recebido em 04/Out/2018

Versão final reapresentada em 16/Jun/2019 Aprovado em 08/Jul/2019 